\title{
IKK $\beta$ regulates essential functions of the vascular endothelium through kinase-dependent and -independent pathways
}

\author{
Noboru Ashida ${ }^{1, \dagger}$, Sucharita SenBanerjee ${ }^{1, \dagger}$, Shohta Kodama ${ }^{2, \uparrow}$, Shi Yin Foo ${ }^{1,3, \uparrow}$, Matthew Coggins ${ }^{1}$, Joel A. Spencer ${ }^{4}$, \\ Parisa Zamiri ${ }^{4,}$, , Dongxiao Shen ${ }^{5}$, Ling Li ${ }^{1}$, Tracey Sciuto ${ }^{6}$, Ann Dvorak ${ }^{6}$, Robert E. Gerszten ${ }^{3,5}$, Charles P. Lin ${ }^{4}$, \\ Michael Karin7 \& Anthony Rosenzweig ${ }^{1}$
}

Vascular endothelium provides a selective barrier between the blood and tissues, participates in wound healing and angiogenesis, and regulates tissue recruitment of inflammatory cells. Nuclear factor (NF)- $\mathrm{kB}$ transcription factors are pivotal regulators of survival and inflammation, and have been suggested as potential therapeutic targets in cancer and inflammatory diseases. Here we show that mice lacking IKK $\beta$, the primary kinase mediating NF- $\mathrm{KB}$ activation, are smaller than littermates and born at less than the expected Mendelian frequency in association with hypotrophic and hypovascular placentae. IKK $\beta$-deleted endothelium manifests increased vascular permeability and reduced migration. Surprisingly, we find that these defects result from loss of kinase-independent effects of IKK $\beta$ on activation of the serine-threonine kinase, Akt. Together, these data demonstrate essential roles for IKK $\beta$ in regulating endothelial permeability and migration, as well as an unanticipated connection between IKK $\beta$ and Akt signalling.

\footnotetext{
${ }^{1}$ The Cardiovascular Institute, Beth Israel Deaconess Medical Center, Harvard Medical School, Boston, Massachusetts 02215, USA. ${ }^{2}$ Tissue Engineering and Regenerative Medicine, Brigham and Women's Hospital, Harvard Medical School, Boston, Massachusetts 02115, USA. ${ }^{3}$ Cardiovascular Division, MGH, Harvard Medical School, Boston, Massachusetts 02114, USA. ${ }^{4}$ Wellman Center for Photomedicine, Massachusetts General Hospital (MGH), Harvard Medical School, Boston, Massachusetts 02114, USA. ${ }^{5}$ Center for Immunology and Inflammatory Diseases MGH, Harvard Medical School, Charlestown, Massachusetts 02129, USA. ${ }^{6}$ Department of Pathology, Beth Israel Deaconess Medical Center, Harvard Medical School, Boston, Massachusetts 02215, USA. 7 Department of Pharmacology, UCSD, San Diego, California 92093, USA. †Present addresses: Department of Clinical Innovative Medicine, Translational Research Center, Kyoto University Faculty of Medicine, Kyoto 606-8507, Japan (N.A.); Department of Gastroenterology, Brigham and Women's Hospital, Harvard Medical School, Boston, Massachusetts 02115, USA (S.S.); Department of Regenerative Medicine and Transplantation, Fukuoka University Faculty of Medicine, Fukuoka 814-0180, Japan (S.K.); Cardiovascular Translational Medicine, Novartis Institute for Biomedical Research, Cambridge, Massachusetts 02139, USA (S.Y.F.); Arsenal Medical, Watertown, Massachusetts 02472, USA (P.Z.). Correspondence and requests for materials should be addressed to A.R. (email: arosenzw@bidmc.harvard.edu) or to N.A. (email: nashida@kuhp.kyoto-u.ac.jp).
} 
$\mathrm{N}$ uclear factor (NF)- $\kappa B$ transcription factors (p65 or RelA, p50, p52, c-Rel, and RelB) generally exist as homo- or heterodimers in the cytosol bound to one of three inhibitory, IKB, subunits (reviewed in ref. 1). In response to a wide variety of stimuli, $\mathrm{NF}-\kappa \mathrm{B}$ is classically activated through serine phosphorylation and degradation of I $\kappa \mathrm{B}$ via the ubiquitin pathway, followed by translocation of NF- $\kappa B$ to the nucleus where it activates transcription. Serine phosphorylation of I $\mathrm{KB}$ is mediated by a large multi-unit complex containing two catalytic subunits (I kappa B kinase (IKK) $\alpha$ and $\mathrm{IKK} \beta)^{2,3}$, as well as the regulatory subunit IKK $\gamma$ or $\mathrm{NEMO}^{4}$. IKK $\alpha$ has an important role in skin development independent of its kinase activity $^{5}$ as well as a specialized role in the alternative pathway of $\mathrm{NF}-\kappa \mathrm{B}$ activation that induces specific genes in B cells via NF- $\kappa \mathrm{B} 2$ and $\mathrm{RelB}^{6}$. IKK $\beta$ appears to be the primary kinase mediating phosphorylation of IKB in most cell types and germline deletion of IKK $\beta$ results in embryonic lethality due to massive liver apoptosis ${ }^{7}$.

In vascular endothelium, NF- $\kappa B$ activation is seen in a wide variety of diseases ranging from atherosclerosis ${ }^{8}$ to cancer $^{1}$. In addition to a role in endothelial inflammation, previous work has suggested a role for NF- $\kappa \mathrm{B}$ in other essential endothelial functions. Pharmacological inhibition of NF- $\mathrm{BB}$ decreases vascular permeability ${ }^{9}$, although a recent report, using Tie2-driven transgenic overexpression of a superinhibitory I $\mathrm{\kappa B}-\alpha(\mathrm{sI} \kappa \mathrm{B})$, demonstrated increased vascular permeability after mustard oil stimulation as well as alterations in endothelial tight junctions ${ }^{10}$. Tie2-sI $\mathrm{KB}$ mice were born at the expected frequency and developed normally despite endothelial NF- $\kappa \mathrm{B}$ inhibition ${ }^{10}$. A role in endothelial migration has not been reported, although IKK $\beta$ deletion promotes migration of embryonic fibroblasts ${ }^{11}$.

To better delineate the role of IKK $\beta$ in mammalian endothelium in vivo, we used conditional inactivation to generate endothelialspecific IKK $\beta$-deleted mice. These studies show essential roles for IKK $\beta$ in the developing placental and adult somatic vasculature, including regulation of endothelial apoptosis and migration, vascular permeability, and vasculogenesis. Surprisingly, although some of these effects (such as apoptosis) result from loss of IKK $\beta$ kinase-dependent activation of NF- $\mathrm{KB}$, others (such as permeability and migration) reflect loss of kinase-independent IKK $\beta$ modulation, the serine/threonine kinase, Akt. Together, these data demonstrate distinct kinase-dependent and -independent roles for IKK $\beta$ in regulating endothelial function, as well as an unanticipated connection between IKK $\beta$ and Akt signalling.

\section{Results}

Generation of endothelial-specific knockout mice. To produce mice with endothelial deletion of IKK $\beta$ (IKK $\beta^{\Delta \mathrm{EC}}$ ), we bred Tie2Cre transgenic mice with IKK $\beta^{\text {flox/flox }}$ mice ${ }^{12}$ on an atherosclerosisprone ApoE-null genetic background. Interestingly, Tie2-Cre ${ }^{+}$ $\mathrm{IKK} \beta^{\text {flox/flox }}-\mathrm{ApoE}^{-/-}$mice were born at lower than the expected Mendelian frequency (7/48 versus $24 / 48$ expected, $P<0.0001$ by chi-square analysis) raising the possibility of prenatal mortality and were $\sim 25 \%$ smaller than Cre-negative littermates at 8 and 32 weeks (Fig. 1a, $P<0.005$ in repeated two-way ANOVA) in contrast to previously published results with Tie2-sIкB mice ${ }^{10}$. Collection of embryos after timed pregnancies revealed that 4/14 (29\%) of the Tie2-Cre ${ }^{+}-\mathrm{IKK} \beta^{\text {flox/flox }}$ embryos on either an $\mathrm{ApoE}^{-/-}$or $\mathrm{ApoE}^{+/-}$ were dead at E14-16 without evident gross malformation of major organs or vascular structures (Fig. 1b) compared with no fetal loss of the Tie2-Cre-negative IKK $\beta^{\text {flox/flox }}$ embryos. Interestingly, placentae from embryos with endothelial IKK $\beta$ deletion were hypomorphic with fewer blood vessels (Fig. 1c,d).

Intact vascular endothelium with altered function. Intact vascular endothelium without detectable endothelial IKK $\beta$ expression in IKK $\beta^{\triangle \mathrm{EC}}$ mice was demonstrated in confocal immunohistochemistry (Fig. 1e) and immunoperoxidase staining (Supplementary
Fig. S1a) of mice after birth. Electron microscopy confirmed the presence of normal-appearing vascular endothelium, and, in contrast to results with transgenic overexpression of $\mathrm{sI \kappa B}^{10}$, normalappearing endothelial tight junctions (Supplementary Fig. S1b-e). To examine the structure and function of IKK $\beta^{\Delta \mathrm{EC}}$ microvessels in vivo, we utilized in vivo microscopy of ear vessels after intravenous injection of Evans blue dye, which binds serum albumen (Fig. 2a; Supplementary Movie 1). Whereas vessel number and branching pattern appeared generally normal, dye rapidly extravasated in the endothelial IKK $\beta$-knockout mice, indicating an increase in baseline vascular permeability even more severe than that seen in Tie2-sIкB mice $^{10}$, as it occurred in the absence of stimulation and contrary to with the reduced permeability reported with pharmacological NF- $\kappa$ B inhibition ${ }^{9}$.

Another important function of vascular endothelium is cellular migration, which has a role in wound healing and angiogenesis. We measured the ability of IKK $\beta$-deficient endothelial cells to migrate in response to the endothelial-specific stimulus, vascular endothelial growth factor (VEGF), in vitro. Endothelial cells from IKK $\beta^{\text {flox/llox }}$ mice were infected with a Cre-expressing adenovirus, which effectively mediated deletion of IKK $\beta$ and inhibited NF- $\kappa B$ activation without affecting IKK- $\alpha$ or $-\gamma$ (Supplementary Fig. S2). Surprisingly, although IKK $\beta$ deletion enhanced fibroblast migration $^{11}$, Cre-mediated deletion of IKK $\beta$ in endothelial cells cultured from IKK $\beta^{\text {flox/flox }}$ mice reduced chemotactic migration to VEGF (Fig. 2b) and migration in a scratch wound-healing assay (Fig. 2c), suggesting a cell-specific role of IKK $\beta$ in regulating cell migration. Deletion of endothelial IKK $\beta$ ex vivo also recapitulated the increased permeability observed in vivo (Fig. 2d). Co-infection of endothelial cells with a recombinant adenovirus encoding wildtype IKK $\beta$, but not a reporter construct, largely restored endothelial migration and reduced permeability to normal (Fig. 2b-d) demonstrating the specificity of this effect. Surprisingly, however, a kinaseinactive mutant of IKK $\beta$ (KD-IKK $\beta$ ), which we had previously shown inhibits endothelial NF- $\kappa B$ activation ${ }^{13}$, also rescued these phenotypes with equal efficacy, suggesting they were not due to loss of kinase activity in the IKK $\beta$ null cells. Kinase assays confirmed that the wild-type IKK $\beta$ construct increased basal IKK $\beta$ kinase activity, which was further increased by cytokine stimulation (Supplementary Fig. S3). In contrast, the KD-IKK $\beta$ construct did not manifest IKK $\beta$ kinase activity at baseline or in response to IL-1 stimulation (Supplementary Fig. S3).

Because NF- $\mathrm{KB}$ modulates cell survival through a variety of anti-apoptotic mechanisms ${ }^{1}$, we examined the effects of IKK $\beta$ deletion on apoptosis in endothelial cells. IKK $\beta$ deletion did not affect apoptosis in endothelial cells in the presence of serum but did result in a significant increase in apoptotic endothelial cells after serum deprivation (Fig. 3). Expression of wild type (WT), but not KD IKK $\beta$ in IKK $\beta$-deleted endothelial cells, mitigated serum starvation-induced apoptosis in endothelial cells (Fig. 3). This result suggests that IKK $\beta$ has both kinase-independent and -dependent effects on important phenotypes of endothelial cells, and reinforces the specificity of results above for migration and permeability.

Altered Akt signalling. The abnormal growth and development as well as the severe vascular leakage seen in IKK $\beta^{\triangle \mathrm{EC}}$ contrasted with results reported with endothelial NF- $\kappa B$ inhibition in Tie2-sI $\mathrm{B}$ transgenics ${ }^{10}$, despite use of the same promoter. These differences in combination with kinase-independent rescue in vitro raised the possibility that IKK $\beta$ modulates baseline endothelial function through NF- $\kappa \mathrm{B}$-independent signalling pathways. Indeed, features of the IKK $\beta^{\triangle \mathrm{EC}}$ mice were reminiscent of those seen in Akt1 null mice, including increased vascular permeability ${ }^{14}$ and decreased endothelial migration ex $v i v o^{14,15}$, as well as prenatal mortality, hypotrophic and hypovascular placentae, and small size (thought in 


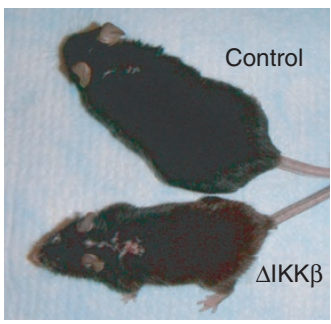

b

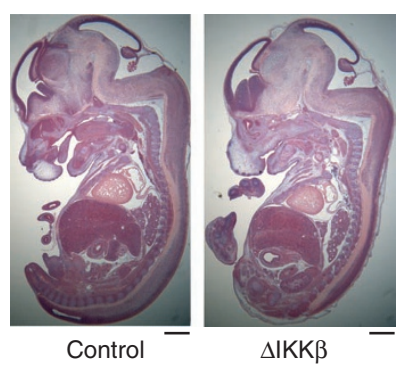

d
C

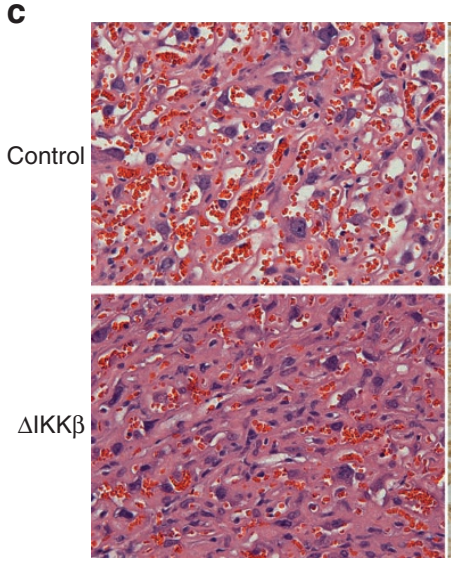

H\&E
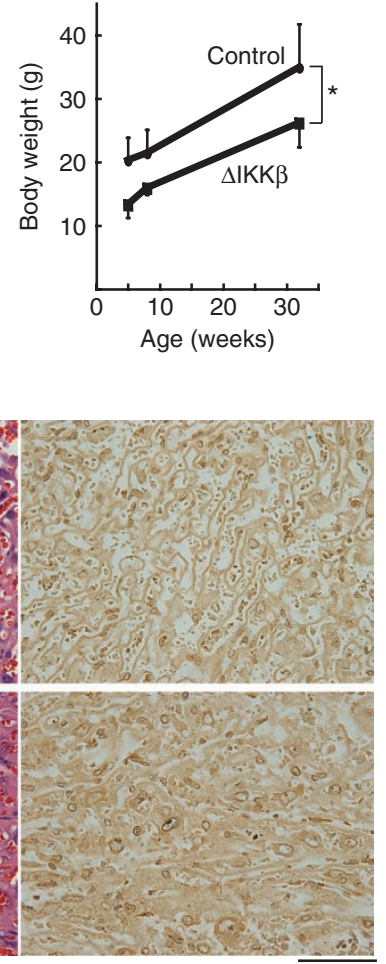

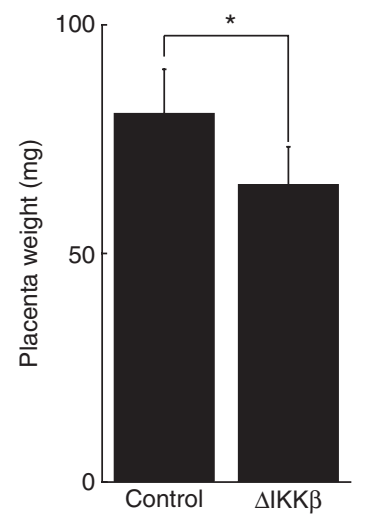

e

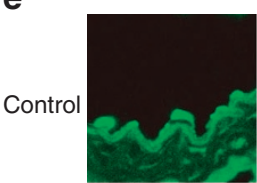

von Willebrand factor

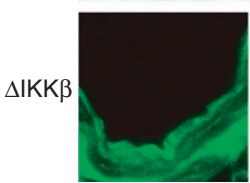

CD146
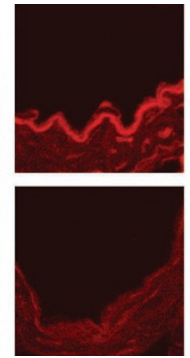

IKK $\beta$
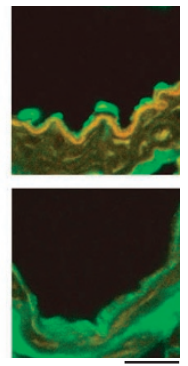

Merge

Figure 1 | Phenotype of endothelial-specific IKK $\beta$ knockout mice. (a) Photograph of littermate wild type ('control') and IKK $\beta^{\triangle E C}$ (' $\triangle I K K \beta^{\prime}$ ) mice and body weight \pm s.d. shown from mice of each genotype at the indicated age $\left(n=3\right.$ /each group, ${ }^{\star} P<0.005$ in repeated two-way ANOVA). (b) H\&E staining of embryos at E14. Scale bars: $1 \mathrm{~mm}$. (c) Staining of placentae by H\&E and immunohistochemistry for von Willebrand Factor at E14. Scale bars: $10 \mu \mathrm{m}$. (d) Placenta weight from mice of each genotype ( $n=6$ in IKK $\beta^{\triangle E C}$ and $n=3$ in control mice, ${ }^{\star} P<0.05$ in two-sided Student's $t$-test. Error bars: \pm s.d.).

(e) Confocal microscopy images of vascular endothelium in IKK ${ }^{\triangle E C}$ and control mice immunostained with antibody for CD146 and IKK 3 . Scale bars: $100 \mu$ m.

Akt1-null mice to reflect reduced Akt1-eNOS signalling in placental vascular endothelium $\left.{ }^{16}\right)$. These similarities and previous reports that IKK isoforms (including IKK $\beta)^{17,18}$ could physically interact with Akt prompted us to examine Akt in IKK $\beta^{\triangle \mathrm{EC}}$ mice. Interestingly, phospho-Akt was markedly reduced in vascular endothelium from IKK $\beta^{\triangle \mathrm{EC}}$ mice (Fig. 4a). Similarly, ex vivo deletion of IKK $\beta$ in endothelial cells resulted in reduced phosphorylation of Akt in response to insulin-like growth factor (IGF)-I (Fig. 4b). A similar blunted response to insulin was seen in IKK $\beta$-deleted endothelial cells in vitro (Supplementary Fig. S4). Phosphorylation of GSK3 $\beta$ in response to insulin was also blunted in IKK $\beta$-deleted endothelial cells, consistent with inhibition of Akt downstream signalling (Supplementary Fig. S5). Conversely, overexpression of either WT or kinase-inactive IKK $\beta$ in vitro was sufficient to increase Akt phosphorylation in endothelial cells (Fig. 4c), consistent with a kinaseindependent effect of IKK $\beta$ on Akt signalling. Previous reports suggested that Akt and IKK $\beta$ physically interact in other cell types ${ }^{18}$.
However, we did not find consistent evidence of robust IKK $\beta$-Akt interaction despite multiple immunoprecipitation, GST-pull down and yeast interaction assays.

Disrupted Akt trafficking. In response to growth factor stimulation, increased phosphatidylinositol $(3,4,5)$-trisphosphate (PIP3) binds the pleckstrin homology domain of Akt and prompts its translocation to the cell membrane, where it is localized in caveolar lipid rafts and phosphorylated, and subsequently moves to the nucleus ${ }^{19}$. Trafficking to lipid rafts has an important role in Akt activation $^{20}$, and nuclear translocation enhances its anti-apoptotic effects $^{21}$. IKK $\beta$ has also been reported to localize to lipid rafts ${ }^{22}$. To examine which step in the activation of Akt is affected by IKK $\beta$ deletion, we examined intracellular Akt trafficking after stimulation with IGF-I in murine endothelial cells in vitro. As previously described ${ }^{19}$, IGF-I stimulation of control cells induced early localization of Akt to the membrane (evident at $5 \mathrm{~min}$ ) followed by movement 


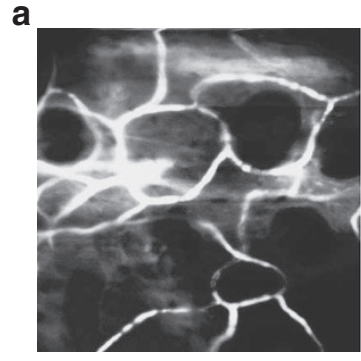

Control

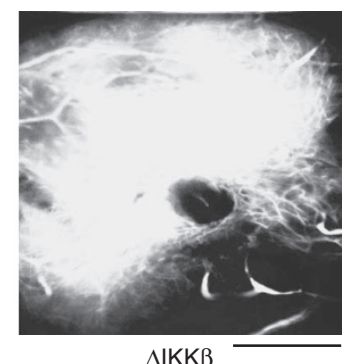

$\Delta \mathrm{IKK} \beta$

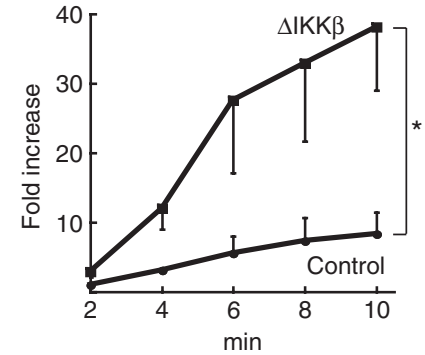

c

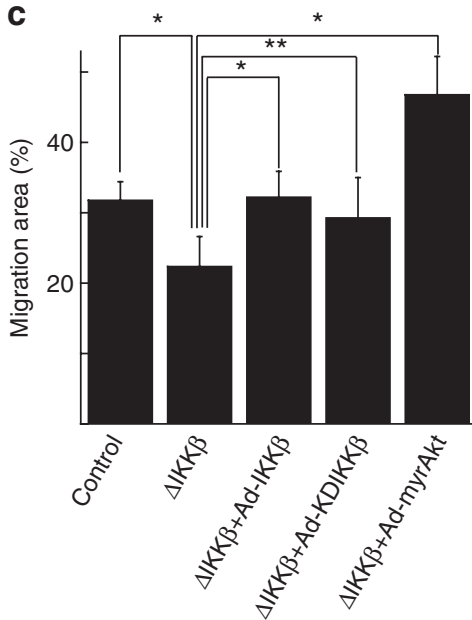

b

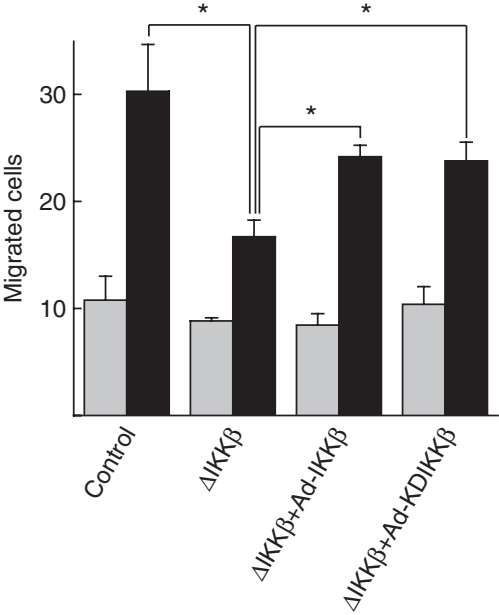

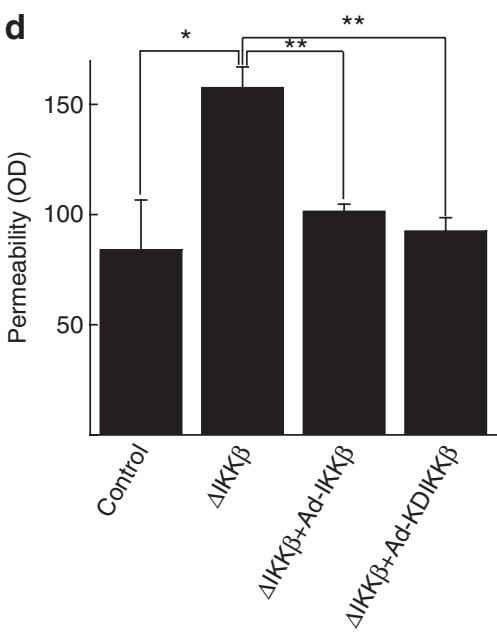

Figure $\mathbf{2}$ | Permeability and migration in IKK $\boldsymbol{\beta}$-manipulated endothelial cells in vivo and in vitro. (a) In vivo microscopy images of ear microvessels after injection of Evans blue dye. Representative image at $10 \mathrm{~min}$ (top) and cumulative data \pm s.e. from three independent experiments (bottom) are shown $\left({ }^{\star} P<0.005\right.$ in repeated two-way ANOVA). Scale bars: $100 \mu \mathrm{m}$. See also Supplementary Movie 1 online. (b) In vitro migration to VEGF of endothelial cells in which IKK $\beta$ is deleted or WT/-IKK $\beta$ is expressed. Cumulative data \pm s.e. from four independent experiments are shown. Grey bars: VEGF ( $)$, black bars: VEGF (+). ${ }^{\star} P<0.05$ in two-sided Student's $t$-test. (c) Wound-healing assay. Migration area was accumulated from six separated observation fields. Representative experiment is shown from three independent experiments total. ${ }^{\star} P<0.005$ in two-sided Student's $t$-test. ${ }^{\star \star} P<0.05$ in two-sided Student's $t$-test. Error bars: \pm s.d. (d) In vitro permeability of endothelial cells in which IKK $\beta$ is deleted or WT/KD-IKK $\beta$ is expressed. Cumulative data \pm s.e. from three independent experiments are shown. ${ }^{\star} P<0.05$ in two-sided Student's $t$-test. ${ }^{\star} P<0.005$ in two-sided Student's $t$-test. OD, optical density.

to the nucleus (at $15 \mathrm{~min}$ ) evident by confocal immunohistochemistry (Supplementary Fig. S6a). Subcellular localization of phosphorylated Akt followed a similar pattern (Supplementary Fig. S6b). In contrast, in endothelial cells in which IKK $\beta$ had been deleted, IGF-I-induced movement of both total and phospho-Akt to the cell membrane at $5 \mathrm{~min}$ and to the nucleus at $15 \mathrm{~min}$ were markedly inhibited (Supplementary Fig. S6a and b). Separation of membrane compartments on a sucrose gradient further documented that IKK $\beta$ deletion reduced Akt in membrane lipid raft fractions after IGF-I stimulation (Fig. 4d). Similarly, subsequent translocation of Akt to the nucleus after IGF-I treatment was inhibited by IKK $\beta$ deletion (Supplementary Fig. S6c). Importantly, endothelial caveolae are present in IKK $\beta^{\triangle \mathrm{EC}}$ mice and appear normal by electron microscopy (Supplementary Fig. S1b-e), suggesting the observed changes 


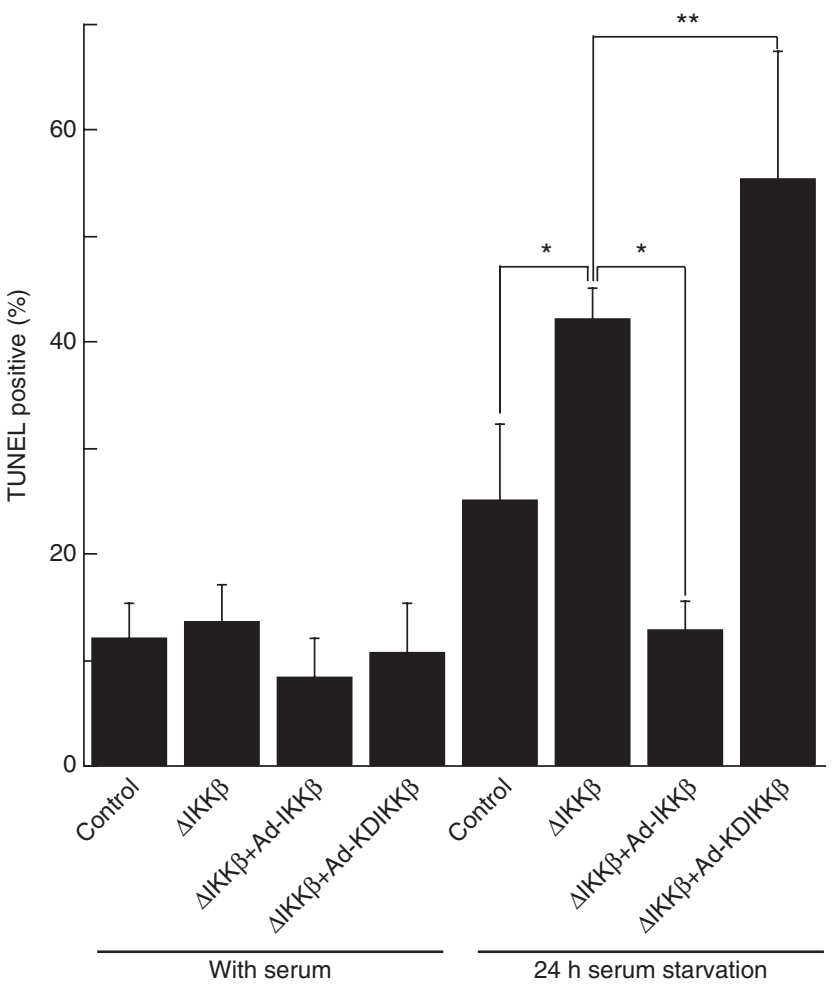

Figure 3 | Effect of IKK $\beta$ on apoptosis induced by $24 \mathrm{~h}$ serum starvation. Primary cultured endothelial cells from lungs of IKK $\beta^{\text {flox/flox }}$ mice with infection of Ad-GFP, Cre, IKK $\beta$ or KDIKK $\beta$ were serum-starved for $24 \mathrm{~h}$. TUNEL positive cells were counted at seven independent fields (mean cell number: $241.5 /$ field). ${ }^{*} P<0.0005$ in two-sided Student's $t$-test, ${ }^{* *} P<0.05$ in two-sided Student's t-test. Error bars: \pm s.d.

in trafficking are not due to disruption of caveolae. Expression of either WT- or KD-IKK $\beta$ restored Akt trafficking to lipid rafts (Fig. 4e), consistent with the functional rescue of permeability and migration mediated by these constructs. Taken together, these data suggest that IKK $\beta$ deletion impairs Akt trafficking and subsequent activation and that the ability of IKK $\beta$ to enhance Akt localization to lipid rafts does not require IKK kinase activity.

Akt rescues altered migration and permeability. We tested the functional relevance of IKK $\beta$ 's modulation of Akt activation in vitro. Of note, Chen et al. ${ }^{14}$ previously demonstrated that permeability in Akt1-deficient endothelial cells is increased in a similar in vitro assay. As noted above, Cre-mediated deletion of IKK $\beta$ increased endothelial permeability and expression of either WT or KD IKK $\beta$, which both restore Akt trafficking (Fig. 4e) and increase Akt phosphorylation (Fig. 4c), reduced endothelial permeability (Fig. 2d). Expression of activated Akt (myr-Akt) also restored permeability to normal in IKK $\beta$-deleted endothelial cells (Fig. 4f). Similarly, expression of activated Akt (as well as either WT or KD IKK $\beta$, Fig. 2b,c) restored the ability of IKK $\beta$-deleted endothelial cells to migrate in response to VEGF (Fig. $4 \mathrm{~g}$ ) and in a scratch wound-healing assay (Fig. 2c) in vitro. Together, these data suggest that deletion of IKK $\beta$ alters endothelial phenotype at least in part through kinaseindependent regulation of Akt activation.

Altered PTEN activity. In the absence of evidence supporting a robust physical interaction between IKK $\beta$ and Akt, we examined the PTEN phosphatase, which cleaves PIP3, removing the primary stimulus for Akt translocation. Inhibitory phosphorylation of PTEN was significantly decreased by IKK $\beta$ deletion in endothelial cells, and conversely, overexpression of KD- or WT-IKK $\beta$ increased PTEN phosphorylation (Fig. 5a-c). These changes would be expected to increase PTEN phosphatase activity (and decrease Akt translocation/phosphorylation) after IKK $\beta$ deletion, and decrease PTEN phosphatase activity in a kinase-independent manner with overexpression. Direct measurement of PTEN phosphatase activity (Fig. 5d) confirmed that this was indeed the case. Taken together, these data implicate PTEN as a mediator of cross-talk between IKK $\beta$ and Akt, most likely through effects on the well-described pathway of PIP3-induced Akt membrane localization.

Impaired ischemia-induced vasculogenesis. The defect in VEGFinduced endothelial migration seen in vitro after IKK $\beta$ deletion raised the possibility that endothelial IKK $\beta$ would important in vasculogenesis or the formation of new blood vessels in adults. Given the small number of homozygous IKK $\beta^{\triangle \mathrm{EC}}$ mice available for study, we addressed this question in heterozygous IKK $\beta^{\triangle \mathrm{EC}}$ mice. After ligation of the common femoral artery, return of collateral flow was markedly impaired in heterozygous IKK $\beta^{\triangle \mathrm{EC}}$ mice (Fig. 6). These data are consistent with our in vitro observations and underscore the potential pathophysiological importance of endothelial IKK $\beta$ in vasculogenesis due to ischemia or potentially other stimuli.

\section{Discussion}

To better delineate the role of IKK $\beta$ in mammalian endothelium, we used conditional inactivation to generate mice with endothelialspecific inaction of IKK $\beta$ (IKK $\left.\beta^{\Delta \mathrm{EC}}\right)$. Gestational loss of IKK $\beta^{\Delta \mathrm{EC}}$ embryos and stunted growth probably resulted from hypomorphic placentae. Late stage embryos and surviving adults manifested an intact somatic vasculature with surprisingly normal morphology. Loss of IKK $\beta$ blocked endothelial NF- $\kappa B$ activation. Whereas endothelial apoptosis was not increased at baseline, IKK $\beta$-deleted endothelial cells were more susceptible to serum deprivationinduced apoptosis in vitro and apoptosis was mitigated by expression of wild-type but not kinase-inactive IKK $\beta$. Adult animals lacking endothelial IKK $\beta$ manifest increased vascular permeability and impaired vasculogenesis in response to ischemia. In vitro, IKK $\beta$-deleted endothelial cells also demonstrated increased permeability and reduced migration. Surprisingly, these defects were rescued equally well by wild-type and kinase-inactive IKK $\beta$ and appear related to kinase-independent activation by IKK $\beta$ of the serine/threonine kinase, Akt. Taken together, these data establish an important physiological role for IKK $\beta$ in regulating a variety of endothelial functions in mammals, and show unanticipated kinase-independent effects of IKK $\beta$ mediating cross-talk with Akt signalling.

The increased vascular permeability seen in vivo in $\mathrm{IKK} \beta^{\Delta \mathrm{EC}}$ mice, is more severe than that seen with either isolated Akt1 deletion $^{14}$ or transgenic endothelial NF- $\kappa \mathrm{B}$ inhibition ${ }^{10}$, probably reflecting the combined effects of endothelial inhibition of both Akt and IKK $\beta-N F-\kappa B$ signalling. This inference is reinforced by the complex relationship of Akt signalling to endothelial permeability. Although Chen et al. ${ }^{10}$ found increased permeability in $\mathrm{Akt}^{-/-}$endothelium both in vivo and in an in vitro model similar to the one used here, Ackah et al. ${ }^{15}$ found reduced vascular permeability in Akt1 ${ }^{-1-}$ mice in response to VEGF. These differences may reflect technical aspects of the assays used and/or the time-points examined. In addition, the IKK $\beta^{\triangle \mathrm{EC}}$ mice studied here differ from the Akt $1^{-1-}$ mice not only in the primary and concomitant loss of endothelial IKK $\beta$ signalling but also in that Akt signalling is preserved in non-endothelial cells, which may be an important source of a variety of factors affecting vascular permeability including VEGF and angiopoietins.

Our data document cross-talk between two central signalling molecules, IKK $\beta$ and Akt, in endothelial cells in vitro and in vivo that appears mediated via the phosphatase, PTEN. Our results differ from previous reports in important respects. First, although 

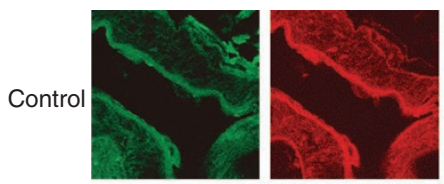

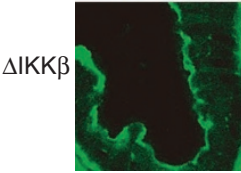

CD146

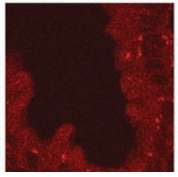

pAkt

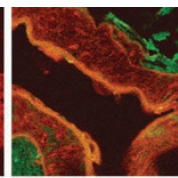

b
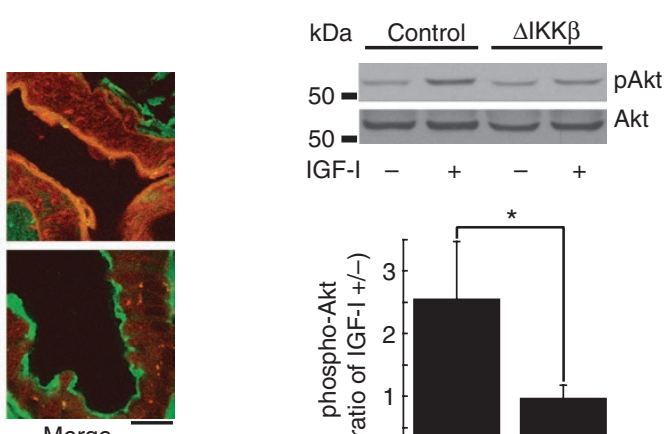

Merge d

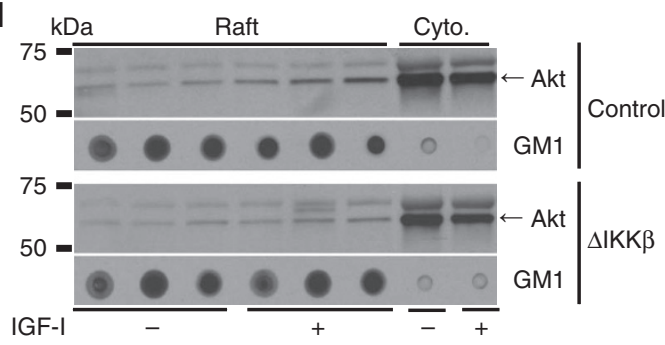

e

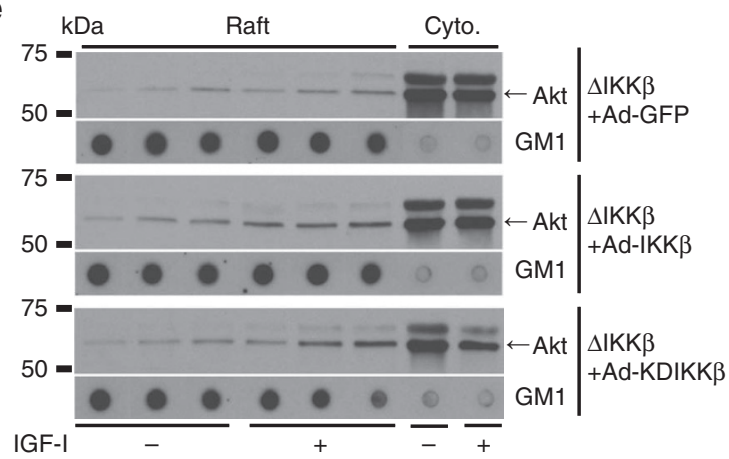

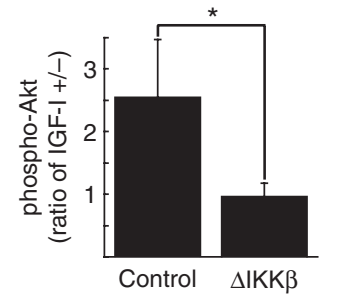

c
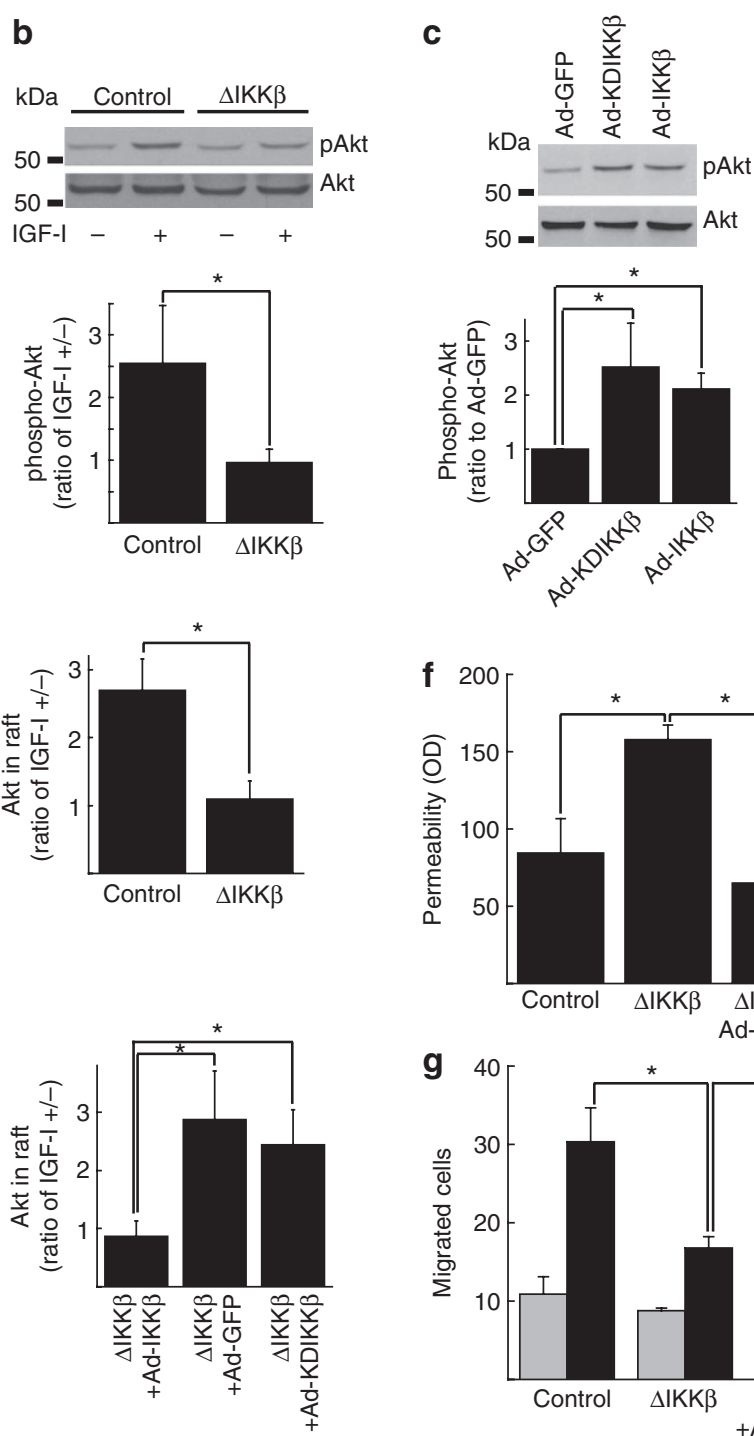
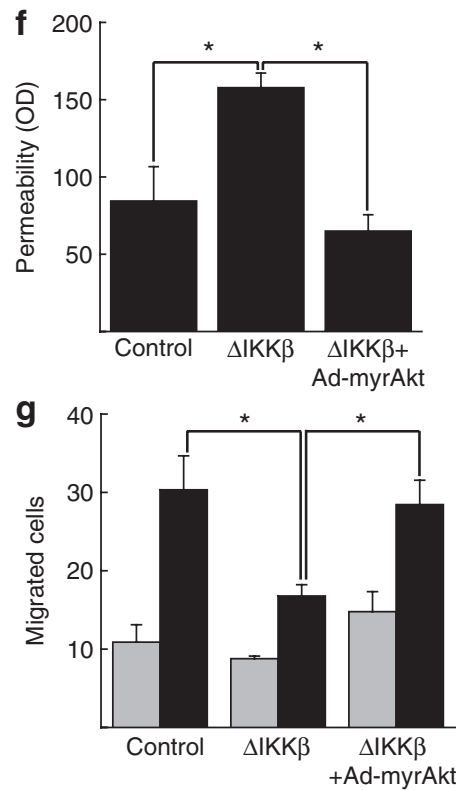

Figure 4 | Akt phosphorylation and localization in IKK $\beta$-manipulated endothelial cells in vivo and in vitro. (a) Confocal microscopy images of endothelial cells in IKK ${ }^{\triangle E C}$ mice immunostained with antibody for CD146 and pAkt. Scale bar: $20 \mu \mathrm{m}$. (b) Representative immunoblots for pAkt and Akt in IKK $\beta$-deleted endothelial cells treated with/without IGF-I and its cumulative quantitative densitometry data \pm s.d. from three independent experiments ( ${ }^{\star} P<0.05$ in two-sided Student's $t$-test). (c) Representative immunoblots for $p A k t$ and Akt in endothelial cells overexpressed with WT- or KD-IKK $\beta$ and its cumulative quantitative densitometry data \pm s.d. from three independent experiments $\left({ }^{\star} P<0.05\right.$ in two-sided Student's $t$-test). (d) Representative Akt immunoblots of fractions from sucrose gradient centrifugation of IKK $\beta$-deleted endothelial cells treated with/without IGF-I and its cumulative quantitative densitometry data \pm s.e. from three independent experiments ( ${ }^{\star} P<0.05$ in two-sided Student's $t$-test). Ganglioside GM1 is marker for lipid raft fractions.

(e) Representative Akt immunoblots of fractions from sucrose gradient centrifugation of IKK $\beta$-deleted endothelial cells expressed with either WT- or KD-IKK $\beta$ treated with/without IGF-I and its cumulative quantitative densitometry data \pm s.e. ( $n=5$ for $\Delta \mathrm{IKK} \beta+\mathrm{Ad}-\mathrm{GFP} ; n=3$ for $\Delta \mathrm{IKK} \beta+\mathrm{Ad}-\mathrm{IKK} \beta$; $n=5$ for $\Delta \mathrm{IKK} \beta+\mathrm{Ad}-\mathrm{KDIKK} \beta$; ${ }^{\star} P<0.05$ in two-sided Student's $t$-test). Ganglioside GM1 is marker for lipid raft fractions. (f, $\left.\mathbf{g}\right)$ In vitro permeability (f) and migration to VEGF ( $\mathbf{g}$ ) in endothelial cells in which IKK $\beta$ is deleted or myr-Akt is expressed. Gray bars: VEGF ( - ), black bars: VEGF (+) for ( $\mathbf{g}$ ). Each panel is cumulative data \pm s.e. from three independent experiments ( ${ }^{\star} P<0.05$ in two-sided Student's $t$-test).

we do find evidence of a signalling connection similar to that described previously ${ }^{17,18,23}$, here we find that Akt lies downstream of IKK $\beta$ (rather than the reverse), and the two molecules work together to exert important effects on baseline endothelial function. In addition, although it is difficult to completely exclude physical interaction as a contributor to these effects, our data suggest that PTEN has an important role as an intermediary. It is not clear whether these kinase-independent effects of IKK $\beta$ work via the IKK signalling complex. The observation that overexpression of IKK $\beta$ at levels nonstoichiometric to other components of the IKK complex increases Akt activation, raises the possibility that IKK $\beta$ acts independent of the complex in this context. In addition, we note that IKK $\alpha$ knockout mice ${ }^{24}$ do not phenocopy any of the features we observed in IKK $\beta$ endothelial knockout mice, further supporting the hypothesis that IKK $\beta$ 's role in this context does not require IKK $\alpha$. However, testing these hypotheses awaits delineation of the precise molecular mechanism by which IKK $\beta$ modulates PTEN activity.

In contrast to our results, several studies in other tissues have reported that IKK $\beta$ activation can inhibit insulin signalling in general and Akt in particular ${ }^{20,25,26}$. These differences may reflect tissue specific roles of IKK $\beta$, similar to the skin-specific effects revealed by IKK $\alpha$ deletion ${ }^{5}$. However, even in other tissues, IKK $\beta$-induced inhibition of Akt signalling was most pronounced in chronic 
a
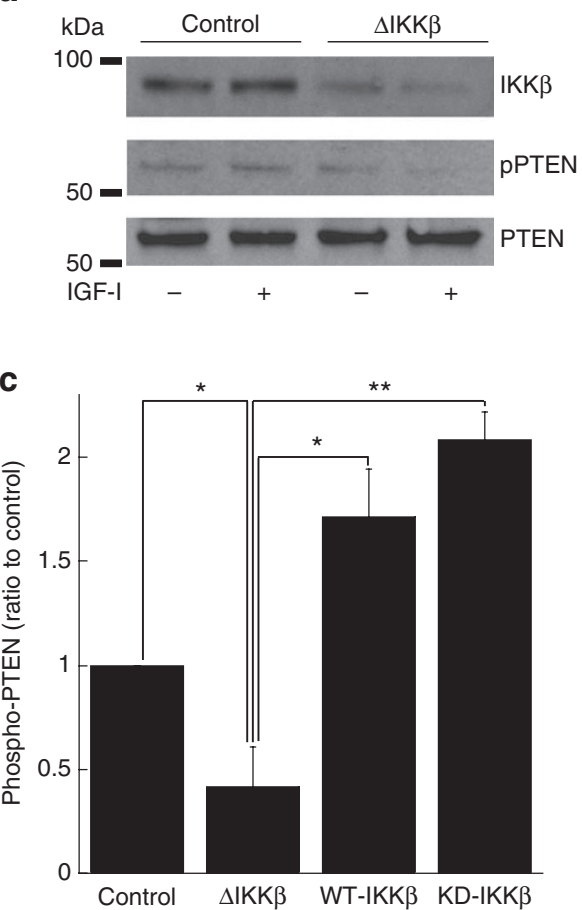

b
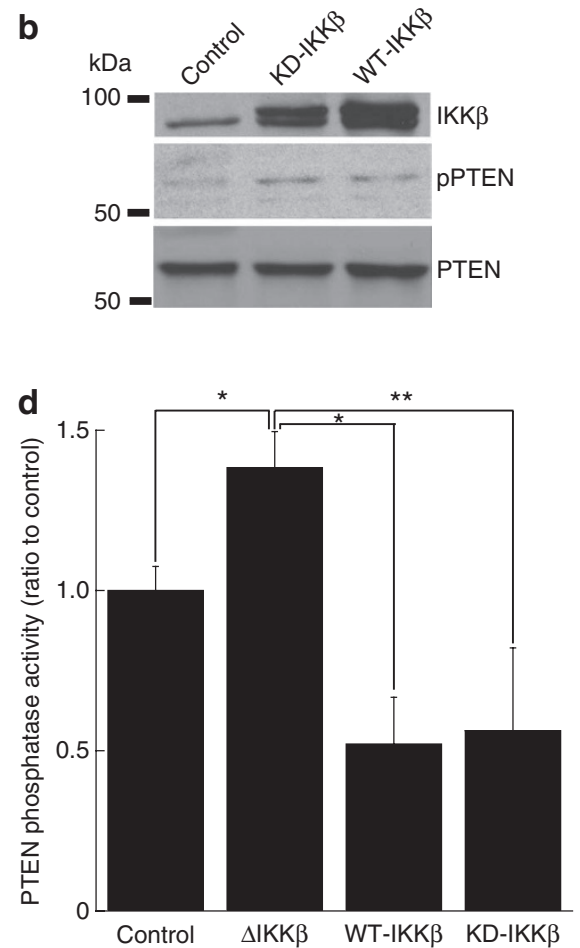

Figure $\mathbf{5}$ | PTEN phosphorylation and phosphatase activity in IKK $\beta$-manipulated endothelial cells. (a-c) Representative immunoblots for IKK $\beta$, pPTEN or PTEN in IKK $\beta$-deleted endothelial cells (a) or WT/KD IKK $\beta$ expressed endothelial cells (b) and its cumulative quantitative densitometry data \pm s.d. from three independent experiments $(\mathbf{c})\left({ }^{\star} P<0.05\right.$ in two-sided Student's $t$-test, ${ }^{\star \star} P<0.0005$ in two-sided Student's $t$-test). (d) Phosphatase activity of PTEN in IKK $\beta$-deleted or WT/KD IKK $\beta$ expressed endothelial cells. Cumulative data \pm s.d. from four independent experiments are shown ( ${ }^{\star} P<0.005$ in two-sided Student's $t$-test, ${ }^{\star \star} P<0.05$ in two-sided Student's $t$-test).
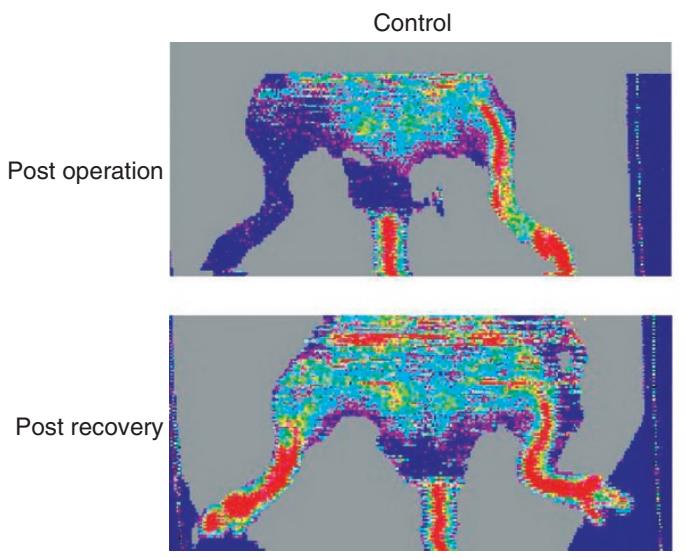
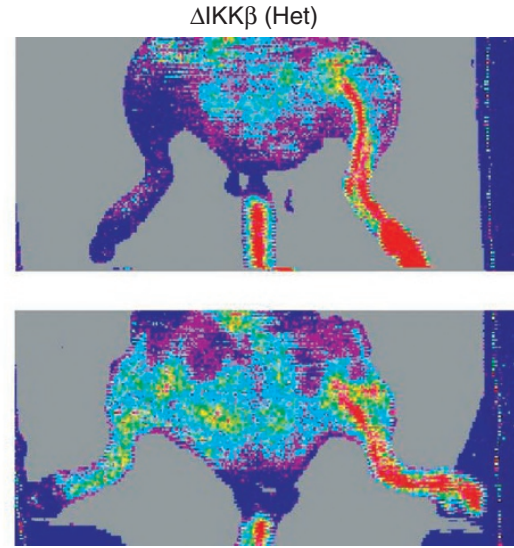

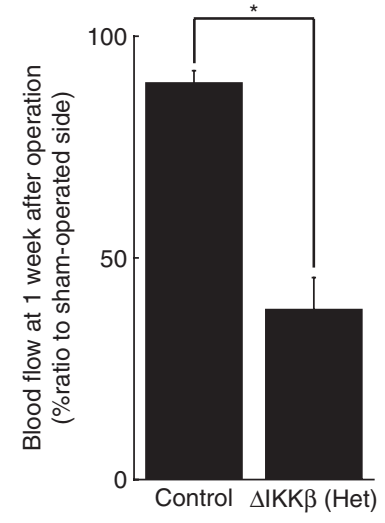

Figure 6 | Ischemia-induced neovascularization in vivo. Representative images of blood flow after ligation of common femoral artery, and quantified cumulative data \pm s.d. from six animals (3 Cre-negative, 3 heterozygous IKK $\beta^{\triangle E C}$ mice. ${ }^{\star} P<0.0005$ in two-sided Student's $t$-test).

inflammatory states or tissues with significant basal NF- $\kappa \mathrm{B}$ activation $^{20,25,26}$. The findings reported here occur in unactivated endothelial cells at baseline. As noted above, we also provide evidence of kinase-dependent effects of IKK $\beta$ on important endothelial phenotypes (for example, apoptosis), although not under basal conditions. Thus, it is quite possible that the relative importance of kinase-dependent and -independent IKK $\beta$ effects would shift, even in endothelial cells, in an inflammatory milieu. Nevertheless, these results broaden our understanding of the complex interactions of the IKK signalosome and demonstrate that IKK $\beta$ has important kinase- and NF- $\kappa \mathrm{B}$-independent effects, similar conceptually to those shown previously for IKK $\alpha^{5}$, in addition to its well-documented kinase-dependent effects.

Most importantly, these data establish an essential role for IKK $\beta$ in fundamental endothelial functions including permeability and migration, and demonstrate the pathophysiological relevance of these effects in a model of ischemia-induced neovascularization. These findings and the observed interaction with Akt signalling may complicate efforts to target IKK $\beta$ in some diseases such as atherosclerosis but could be advantageous in other settings. For example, recent work suggests sustained endothelial Akt activation is necessary for maintenance of abnormal tumour vasculature ${ }^{27}$. 
As IKK $\beta$ is also a potential target for cancer therapy ${ }^{1}$, strategies aimed at decreasing IKK $\beta$ expression could provide additional benefit through secondary inhibition of endothelial Akt signalling and potentially through inhibition of angiogenesis. Understanding the role of IKK $\beta$ in vascular endothelium and the associated signalling cascades could help guide therapeutic strategies for a variety of conditions marked by endothelial activation and dysfunction.

\section{Methods}

Antibodies and reagents. Antibodies used include monoclonal anti-IKK $\beta$ (Upstate), polyclonal anti-IKK $\beta$ (H-470, Santa Cruz Biotechnology), anti-CD146 (Chemicon), anti-total and phospho-(Ser473)Akt, total and phospho-(Ser380/ Thr382/383)PTEN, phospho-GSK3 $\alpha / \beta$ (Cell Signaling). Catch \& Release Immunoprecipitation kit (Upstate), ApopTag Red Apoptosis detection kit (Chemicon), NE-PER Nuclear and Cytoplasmic Extraction Reagents Kit (Pierce) and Cell Lysis Buffer (Cell Signaling) were used in accordance with the manufacturers' instructions. Adenoviral vectors encoding WT IKK $\beta$, kinase-defective (KD) IKK $\beta$, myrAkt, EGFP, Cre and LacZ have been described previously in detail ${ }^{13,28}$.

Animal studies. All animal studies were performed after institutional approval and in compliance with applicable guidelines for animal experimentation. IKK $\beta^{\text {flox } f \text { lox }}$ mice were mated with $\mathrm{ApoE}^{-1-}$ (Jackson Immunoresearch Laboratories) to generate IKK $\beta^{\text {flox } f \text { lox }} \mathrm{ApoE}^{-1-}$ mice which were mated with male Tie2-Cre mice to generate ultimately Tie2-Cre ${ }^{+}-\mathrm{IKK}^{\text {flox/flox}}-\mathrm{ApoE}^{-1-}$ or Tie2-Cre ${ }^{+}-\mathrm{IKK}^{\text {flox/flox}}-$ $\mathrm{ApoE}^{+/-}$mice. As the phenotype appeared identical on the $\mathrm{ApoE}^{-1-}$ or ${ }^{+/-}$background, data from these mice was combined in some studies.

Immunohistochemistry. Slides were incubated in $80 \%$ acetone $\left(2 \mathrm{~min}, 4^{\circ} \mathrm{C}\right)$ and incubated in $3 \%$ hydrogen peroxide for $10 \mathrm{~min}$. After incubation for $60 \mathrm{~min}$, in blocking solution (5\% goat serum, $5 \%$ donkey serum in tris buffered saline (TBS)), slides were incubated with diluted antibody for pAkt (1:50), polyclonal $\operatorname{IKK} \beta(1: 100)$ and CD146 (1:100) overnight at $4^{\circ} \mathrm{C}$. Diluted FITC-conjugated goat anti-mouse and rhodamine-conjugated donkey anti-rabbit secondary antibodies (Jackson, 1:100) were applied to the slides for $60 \mathrm{~min}$.

Immunoperoxidase staining. Slides were incubated with Avidin Block (Vector Laboratories SP-2001, Avidin-Biotin blocking kit) for $15 \mathrm{~min}$ before applying Biotin Block for fifteen minutes. Diluted rabbit anti-Von Willibrand Factor (Chemicon, 1:200) was applied to each slide $\left(4^{\circ} \mathrm{C}\right.$, overnight). Diluted biotinylated donkey anti-rabbit secondary antibody (Jackson, 1:200) was applied for $60 \mathrm{~min}$ at room temperature. $\mathrm{ABC}$ reagent (Vector) was applied to slides for $30 \mathrm{~min}$ at room temperature. DAB solution (diluted 1:10 in TBS, $\mathrm{pH}$ 7.6) added with $3 \%$ hydrogen peroxide (1:125) was applied to slides for approximately $1 \mathrm{~min}$. Slides were then counterstained with hematoxylin.

In vivo permeability assay. Vessel permeability was assessed by leakage of the Evans blue dye from the mouse ear vasculature following intravenous injection. An intravenous catheter was inserted into the tail vein of anaesthetized mouse before placing the mouse on the stage of a video-rate scanning laser confocal microscope. The ear skin was gently spread onto a coverglass with a drop of $1 \%$ methocellulose. At the start of each experiment, $100 \mu \mathrm{l}$ of $2 \%$ Evan's blue dye was injected via the tail vein. Images were acquired from an appropriate vascular area (at about $50 \mu \mathrm{m}$ under the surface of the skin) and every $30 \mathrm{~s}$ for at least $10 \mathrm{~min}$ after injection. Each image was acquired by averaging 30 video frames ( 1 s integration time) using an Olympus 60×, numerical aperture 1.2, water-immersion objective producing optical sectioning images with sub-micron resolution comprising $500 \times 500$ pixels in a $300 \mu \mathrm{m} \times 300 \mu \mathrm{m}$ field of view. Evan's blue dye was excited by a $635 \mathrm{~nm}$ laser (Edmund Optics) and fluorescence detected through a $695 \pm 27.5 \mathrm{~nm}$ bandpass filter (Omega Optical) by a photo multiplier tube (Hamamatsu). To determine vessel permeability, acquired images (as a function of time) were first co-registered and then put in ImageJ as a stack. Using the polygonal selection tool, the change in fluorescence intensity was recorded in 4-8 tissues areas adjacent to blood vessel per stack. The fold increase of each transgenic animal $(n=3)$ was normalized to its littermate control at $2 \mathrm{~min}$ after injection and results were pooled.

Endothelial cell culture. Lungs of IKK $\beta^{\text {fox } f \text { flox }}$ mice were harvested, minced finely and digested in $25 \mathrm{ml}$ collagenase (Roche, $2 \mathrm{mg} \mathrm{ml}^{-1}$ ). Cells were incubated with anti-PECAM-1 antibody (BD Biosciences, 1:100) and cells bound with antibody were isolated magnetically with mouse anti-rat kappa microbead (Miltenyi Biotec) washed with complete media (DMEM, 20\% FCS, $0.1 \mathrm{mg} \mathrm{ml}^{-1}$ heparin, $0.1 \mathrm{mg} \mathrm{ml}^{-1}$ endothelial cell mitogen (Biomedical Technologies), nonessential amino acids, sodium pyruvate, L-glutamine and penicillin/streptomycin at standard concentrations) and cultured in fibronectin-coated, tissue-culture flasks. Nonadherent cells were removed after $24 \mathrm{~h}$ and media replaced. At confluence, endothelial cells were magnetically selected further using anti-ICAM-2 antibody (BD Biosciences, 1:100).

Endothelial migration assay. Endothelial cells were suspended at $5 \times 10^{5} \mathrm{cells}^{-1}$ and $100 \mu \mathrm{l}$ were added to the top chamber of a fibronectin-coated transwell apparatus (6.5-mm diameter, $8-\mu \mathrm{m}$ pore size Costar, Corning Incorporated) whose lower chamber was filled with $600 \mu \mathrm{l}$ DMEM containing $50 \mathrm{ng} \mathrm{ml}^{-1}$ VEGF where indicated (Pepro Tech). Cells were incubated for $6 \mathrm{~h}$ at $37^{\circ} \mathrm{C}$ in an atmosphere containing $5 \% \mathrm{CO}_{2}$, and then fixed with ice-cold methanol for $20 \mathrm{~min}$. Migrated cells were counted after staining with Hema3 Manual Staining System (Fisher Scientific $)^{29}$.

In vitro permeability assay. Permeability assay was performed according to product manual (In vitro Vascular Permeability Assay from Millipore: ECM640). Endothelial cells monolayer forms on collagen-coated inserts which contain $1.0 \mu \mathrm{m}$ symmetrical pores within a transparent polyethylene membrane. $150 \mu \mathrm{l}$ of cell culture medium containing 1:40 FITC-Dextran is added on the top of the cells and incubated at $37^{\circ} \mathrm{C}$ for three hours. The extent of permeability is determined by measuring the fluorescence of the plate well solution using excitation/emission wavelength of $485 \mathrm{~nm} / 530 \mathrm{~nm}$.

Wound-healing assay. Confluent endothelial monolayers in $6 \mathrm{~cm}$ dishes were scraped with sterilized $1 \mathrm{ml}$ micropipette tips to create a cell-free zone. Microscopic images were captured at 0 and $36 \mathrm{~h}$ later, and the percent of the migrated area was quantified using Image J software (National Institutes of Health, USA).

Sucrose gradient. Endothelial cells were infected with the indicated adenoviral vectors, treated with $10 \mathrm{nM}$ IGF-I or PBS for $15 \mathrm{~min}$, and lysed in cell lysis buffer (Cell Signaling ) containing $1 \mathrm{mM}$ phenylmethylsulfonyl fluoride (PMSF). An identical amount of protein from each sample was mixed with $60 \%$ sucrose in $\mathrm{PBS} / \mathrm{MBS}$ buffer $3 \mathrm{ml}$ (final volume, sucrose concentration 51.7-58.7\%), and transferred to a Beckman Ultracentrifuge tube. $3 \mathrm{ml}$ of $35 \%$ sucrose followed by $4 \mathrm{ml}$ of $5 \%$ sucrose were overlaid. Samples were ultracentrifuged (38,000 r.p.m. $\cong 180,000 \times g$ in SW41 rotor (Beckman Coulter), 20 h) and 24 fractions were collected from the top of the gradient. For detection of lipid raft fractions, all fractions were dot-blotted with HRP-labeled cholera toxin B (Sigma) to detect Ganglioside GM1, and raft fractions were immunoblotted for caveolin-1 (BD biosciences) for confirmation.

PTEN activity assay. PTEN activity assay was performed according to product manual (PTEN Activity Assay from Echelon: K-2300). Immunoprecipitated PTEN beads were washed with cell lysis buffer twice and with PTEN reaction buffer (10 mM Hepes, $150 \mathrm{mM} \mathrm{NaCl}, 10 \mathrm{mM}$ DTT, ph 7.2) once, and re-suspended with $300 \mu \mathrm{l}$ reaction buffer. $100 \mu \mathrm{l}$ sample was added to each well of PI(3,4,5)P3-coated PTEN substrate plate, and plate was incubated for $90 \mathrm{~min}$ at $37^{\circ} \mathrm{C}$. Each well was washed with $200 \mu \mathrm{l}$ of TBS-T three times, and PI(4,5)P2 detector was added to the well. Plate was incubated for $1 \mathrm{~h}$ at room temperature, and washed with $200 \mu \mathrm{l}$ of TBS-T three times. $100 \mu \mathrm{l}$ of HRP conjugate working solution prepared by diluting HRP conjugate 1:40 with TBS-GT was added to each well, and plate was incubated for $1 \mathrm{~h}$ at room temperature. After washing with $200 \mu \mathrm{l}$ of TBS-T three times, $100 \mu \mathrm{l}$ of TMB (Cell Signaling) was added to each well and plate was incubated for $30 \mathrm{~min}$ at room temperature. Reaction was stopped with $50 \mu \mathrm{l}$ per well of stop solution (Cell Signaling) and absorbance was read at $450 \mathrm{~nm}$. The values were compared with the standard curve to quantify conversion of $\mathrm{PI}(3,4,5) \mathrm{P} 3$ substrate to $\mathrm{PI}(4,5) \mathrm{P} 2$.

Femoral artery ligation. The epigastric and deep femoral arteries were cauterized. Proximal and distal sutures were placed around the femoral artery which was then cut between the ligatures. Immediately and one week after surgery, hindlimb blood flow was assessed using laser doppler imaging (LDI-2, Moor Inc). Flux, correlating to vascular limb perfusion, was quantitated using the Moor LDI-Main V51 software, and expressed as a ratio of the flux in the ischemic limb to that in the unoperated, nonischemic limb.

Statistical analysis. Unless otherwise specified in the text, statistical comparisons were made with two-sided Student's $t$-test.

\section{References}

1. Karin, M. Nuclear factor-kappaB in cancer development and progression. Nature 441, 431-436 (2006).

2. Zandi, E., Rothwarf, D. M., Delhase, M., Hayakawa, M. \& Karin, M. The IkappaB kinase complex (IKK) contains two kinase subunits, IKKalpha and IKKbeta, necessary for IkappaB phosphorylation and NF-kappaB activation. Cell 91, 243-252 (1997).

3. Mercurio, F. et al. IKK-1 and IKK-2: cytokine-activated IkappaB kinases essential for NF- kappaB activation. Science 278, 860-866 (1997).

4. Rothwarf, D. M., Zandi, E., Natoli, G. \& Karin, M. IKK-gamma is an essential regulatory subunit of the IkappaB kinase complex. Nature 395, 297-300 (1998)

5. Hu, Y. et al. IKKalpha controls formation of the epidermis independently of NF-kappaB. Nature 410, 710-714 (2001).

6. Senftleben, U. et al. Activation by IKKalpha of a second, evolutionary conserved, NF-kappa B signaling pathway. Science 293, 1495-1499 (2001).

7. Li, Z. W. et al. The IKKbeta subunit of IkappaB kinase (IKK) is essential for nuclear factor kappaB activation and prevention of apoptosis. J. Exp. Med. 189, 1839-1845 (1999). 
8. Brand, K. et al. Activated transcription factor nuclear factor-kappa B is present in the atherosclerotic lesion. J. Clin. Invest. 97, 1715-1722 (1996).

9. Liu, S. F., Ye, X. \& Malik, A. B. Pyrrolidine dithiocarbamate prevents I-kappaB degradation and reduces microvascular injury induced by lipopolysaccharide in multiple organs. Mol. Pharmacol. 55, 658-667 (1999).

10. Kisseleva, T. et al. NF-kappaB regulation of endothelial cell function during LPS-induced toxemia and cancer. J. Clin. Invest. 116, 2955-2963 (2006).

11. Chen, F., Lu, Y., Castranova, V., Li, Z. \& Karin, M. Loss of Ikkbeta promotes migration and proliferation of mouse embryo fibroblast cells. J. Biol. Chem. 281, 37142-37149 (2006).

12. Chen, L. W. et al. The two faces of IKK and NF-kappaB inhibition: prevention of systemic inflammation but increased local injury following intestinal ischemia-reperfusion. Nat. Med. 9, 575-581 (2003).

13. Meiler, S. E. et al. Endothelial IKK beta signaling is required for monocyte adhesion under laminar flow conditions. J. Mol. Cell Cardiol. 34, 349-359 (2002).

14. Chen, J. et al. Akt1 regulates pathological angiogenesis, vascular maturation and permeability in vivo. Nat. Med. 11, 1188-1196 (2005)

15. Ackah, E. et al. Akt1/protein kinase Balpha is critical for ischemic and VEGF-mediated angiogenesis. J. Clin. Invest. 115, 2119-2127 (2005).

16. Yang, Z. Z. et al. Protein kinase B alpha/Akt1 regulates placental development and fetal growth. J. Biol. Chem. 278, 32124-32131 (2003).

17. Ozes, O. N. et al. NF-kappaB activation by tumour necrosis factor requires the Akt serine- threonine kinase. Nature 401, 82-85 (1999).

18. Romashkova, J. A. \& Makarov, S. S. NF-kappaB is a target of AKT in antiapoptotic PDGF signalling. Nature 401, 86-90 (1999).

19. Andjelkovic, M. et al. Role of translocation in the activation and function of protein kinase B. J. Biol. Chem. 272, 31515-31524 (1997).

20. Mourkioti, F. et al. Targeted ablation of IKK2 improves skeletal muscle strength, maintains mass, and promotes regeneration. J. Clin. Invest. 116, 2945-2954 (2006)

21. Shiraishi, I. et al. Nuclear targeting of Akt enhances kinase activity and survival of cardiomyocytes. Circ. Res. 94, 884-891 (2004).

22. Su, T. T. et al. PKC-beta controls I kappa B kinase lipid raft recruitment and activation in response to BCR signaling. Nat. Immunol. 3, 780-786 (2002).

23. Zhou, B. P. et al. HER-2/neu blocks tumor necrosis factor-induced apoptosis via the Akt/NF-kappaB pathway. J. Biol. Chem. 275, 8027-8031 (2000).

24. Takeda, K. et al. Limb and skin abnormalities in mice lacking IKKalpha. Science 284, 313-316 (1999)

25. Arkan, M. C. et al. IKK-beta links inflammation to obesity-induced insulin resistance. Nat. Med. 11, 191-198 (2005).

26. Cai, D. et al. Local and systemic insulin resistance resulting from hepatic activation of IKK-beta and NF-kappaB. Nat. Med. 11, 183-190 (2005)
27. Phung, T. L. et al. Pathological angiogenesis is induced by sustained Akt signaling and inhibited by rapamycin. Cancer Cell 10, 159-170 (2006).

28. Matsui, T. et al. Adenoviral gene transfer of activated PI 3-kinase and Akt inhibits apoptosis of hypoxic cardiomyocytes in vitro. Circulation 100, 2373-2379 (1999).

29. Heller, E. A. et al. Inhibition of atherogenesis in BLT1-deficient mice reveals a role for LTB4 and BLT1 in smooth muscle cell recruitment. Circulation 112 578-586 (2005).

\section{Acknowledgments}

This work was supported in part by grants from the NIH (A.R., M.K., R.E.G.) and Leducq Foundation Network of Research Excellence. In addition, N.A. gratefully acknowledge funding from the Sumitomo Life Social Welfare Services Foundation, the Cell Science Research Foundation, The Uehara Memorial Foundation, and Otsuka Pharmaceutical Co., Ltd. We also thank Dr. Serafima Zaltsman for expert management of our mouse colonies and Dr. Harold Dvorak for his insightful discussion of vascular permeability.

\section{Author contributions}

N.A. and A.R. conceived and designed the research. N.A., S.S., M.C., and L.L. performed the experiments. S.K. established primary culture system of endothelial cells and performed embryo experiments. S.Y.F performed ischemia-induced vasculogenesis experiments. J.S., P.Z., and C.L. performed in vivo permeability assay. D.S. and R.G. performed endothelial migration assay. T.S. and A.D. performed electron microscopy imaging. M.K. provided IKK $\beta^{\text {tox } / \text { flox }}$ mice and provided input into the experimental design and interpretation.

\section{Additional information}

Supplementary Information accompanies this paper at http://www.nature.com/ naturecommunications

Competing financial interests: The authors declare no competing financial interests.

Reprints and permission information is available online at http://npg.nature.com/ reprintsandpermissions/

How to cite this article: Ashida, N. et al. IKK $\beta$ regulates essential functions of the vascular endothelium through kinase-dependent and -independent pathways. Nat. Commun. 2:318 doi: 10.1038/ncomms1317 (2011).

License: This work is licensed under a Creative Commons Attribution-NonCommercialNoDerivative Works 3.0 Unported License. To view a copy of this license, visit http:// creativecommons.org/licenses/by-nc-nd/3.0/ 